\title{
Visual Interfaces Designed for Searching Text Content on Mobile Devices
}

\author{
Todd Welch, Gregory Short, and Beomjin Kim
}

\begin{abstract}
The widespread availability of mobile devices has made information available to many on the go, making it a valuable tool in everyday life. Their ability to look up information on the fly and assist with a user's information retrieval in a timely fashion make them a natural everyday carry. However, a limitation of these devices is the lack of screen space available to display information, thus presenting a potential problem to this seemingly ideal device. A technique to present a large amount of information, cohesively, is required to make users' search activities more convenient and effective. We present visualization techniques designed for presenting lengthy text-based documents on mobile devices. The system uses two different visualizations, Overview and Detail. The Overview shows the overall profile of search terms in the document(s). The Detail View presents the requested information, clearly displaying to the user what content is available. By utilizing this two step approach, we can assist users' search activities by making them more effective in their search concerning lengthy text contents. A pilot experiment was conducted to evaluate the effectiveness of these visual interfaces compared to a traditional text-based interface. For a majority of measured items, the testers showed a higher degree of satisfaction using the visualization, that was developed to address the difficulties in exploring text content on a small screen device. Although a larger scale usability test is expected in the near future, our initial study shows the feasibility of using multi-level visual interfaces as a search-supporting tool to review lengthy text documents on mobile devices.
\end{abstract}

Index Terms-Visual interfaces, visualization, mobile devices, text content search.

\section{INTRODUCTION}

The rapid progression of mobile technology has produced multiple devices that are readily available to consumers today. Smartphones today have a vast reach in the global market due to their widespread popularity. Their usefulness is derived not only from their ability to facilitate communication, but also their ability to allow users to search and access information on the go at any desired time. A natural limitation of this technology is the result of the tradeoff of size for portability; the resulting screen space is thus limited. Additionally, lower transmission speeds result in difficulty loading large amounts of information at once, resulting in a higher access cost for data. For example, many web sites have a customized version utilized specifically to cater to these users, addressing these limitations.

Manuscript received April 10, 2015; revised September 8, 2015.

The authors are with the Department of Computer Science, Indiana University-Purdue University Fort Wayne, USA (e-mail: welctb01@students.ipfw.edu, shorgd01@students.ipfw.edu, kimb@ipfw.edu).
Many researchers have introduced various visualization techniques to display a large amount of data on a limited screen space. These compact, but informative, visual illustrations assist users to review presented information intuitively and effectively.

Kim et al. implemented a technique to present visually abstracted web contents utilizing mobile devices [1]. The proposed method categorized different elements of a webpage (image, text, hyperlinks, objects) and then instead of displaying the content itself, displayed color-coded icons in their corresponding locations. Through this visual transformation, it was possible to display a preview of a webpage while still presenting the user with an overview of the content; which reduced the amount of data transmission to the mobile device.Roto et al. also designed a technique to better visualize web pages on mobile devices [2]. Their work was primarily concerned with scaling a large web page to try to optimize the amount of information that could be displayed on a small screen, without losing any information.

Cuttone et al. presented methods for visualizing social interactions and a user's movement geographically on mobile devices [3]. Correia et al. presented visualizations displaying users' personal routines, as well as making inferences about their potential future routes [4]. Chen et al. implemented tools for assisting members of a software project with their collaboration utilizing visualizations [5]. Zhang et al. implemented a technique for viewing stock market information on mobile devices [6]. This was done by utilizing simple scatter plots, bar graphs, and line graphs.

Huot et al. presented a technique for visualization on a mobile device named Spiralist. The technique was primarily concerned with displaying a large list all at once on a small screen, with the circular shape of the list complimenting natural thumb movement for optimal usage [7].Tseng et al. designed a visual abstraction for websites, with the ultimate goal of being a universal abstraction of common attributes that is able to be understood regardless of the language spoken by the user [8]. Abubakar et al. designed an application that presented a 3D view for map navigation, contrasting with more commonly used 2D map approaches [9].

Although these were effective approaches in their corresponding areas, we were unable to find any specifically designed techniques for visualizing long text based information, such as manuals or handbooks, for mobile devices. These materials are difficult to read on small screens, and thus their usage can be encumbered. The increased availability of mobile devices, as well as the increased number of services that are available with them, results in increased usage and demand for information retrieval on the 
go. A technique to assist users to skim through lengthy text-based content, utilizing a limited amount of screen space, is needed in order to optimize the potential of these devices.

The main goal of our study is to develop visualizations that can present moderate to long length documents on mobile devices effectively, utilizing visual abstraction, to assist users to pinpoint the location of their desired information contained within them. We developed a visualization system named Multi-Tiered Visual Interfaces (MVI) that utilizes an Overview + Detail approach to present the graphical illustrations of multiple text-based contents. The overview visualization allows users to compare multiple text-based resources and intuitively locate portions of content that may include user's interest. The Detail visual interface presents underlying profile of selected subareas of documents. By presenting a large amount of text content through two levels of visual abstractions, we expect that the MVI system will enhance user's search activities utilizing mobile devices, which are equipped with small screens, while preventing users from incurring information overload.

\section{METHOD}

Our approach utilizes two different, but connected, visualizations to succinctly display the large amount of information available. The Overview is responsible for showing the user the overall structure of the text-based document, where the desired information is located, as well as how much of the text contains the desired query. After selecting an area of interest from the visualized overview, the user is then presented with the Detail View, which shows them what information is contained within that selected section. This can allow users to locate relevant portions that are worthwhile to review further. Through a combination of both of these techniques it is possible to analyze particular sections, as well as get a general idea of what content is contained within the referenced documents.

\section{A. Index Generation}

The MVI system utilizes information from a table of contents and an index, for each respective data, in order to generate the visual abstraction. Some resources already come with an index, in which case it will be used as is. For documents that are not indexed, a generic index will be generated to serve in place of a man-made one. To do this we would parse the digitized text for all unique terms and generate a traditional index from this. The table of contents can be built based on chapters or sections in the document, if this is not available, then it can also be generated, without knowing specific boundaries, by segmenting the content based on the number of sentences or words using predefined sizes. However; the main purpose of this study is for the development of visualization techniques. Thus, for this study, we chose mid-size text based documents that already had an index and a table of contents, in particular: student handbooks.

\section{B. Overview}

The Overview presents the user with a visualization that concisely shows the distribution of queried information, as well as the frequency of occurrence for multiple search terms. Also shown is the length and structure of the document. This allows the user to identify areas with related information relatively quickly.

Each section of the text-based resources is represented as a black bar of varying length, the length of which is based off of the length of the corresponding section. These bars are then arrayed, in order, with small gaps between them to separate them. These visual components combined convey the length and structure of any given document [10]. Multiple bars can be viewed simultaneously, helping to facilitate comparison and make it easier to determine which is more relevant to the user's query (reference Fig. 1).

RGB color components are used to represent the information that is being queried. Three query boxes are provided, each one corresponding to a primary color, and this search information is then displayed upon the entire array of bars. The intensity of each color is based off of how frequently the term appears within a certain section, with more corresponding text content in a section resulting in a higher intensity for the corresponding color in that section. If two or more terms share the same pages or appear within a close proximity of each other, their color components are then mixed together to present the user with the information that those terms are co-located at a particular point. The result of combining these concepts is shown in Fig. 1.

If any of the search materials have the information the user is searching for, they can select the sections that most interest them from a particular document. Then, by selecting the Detail button after doing so, they will transition to the Detail View which will display the underlying content.

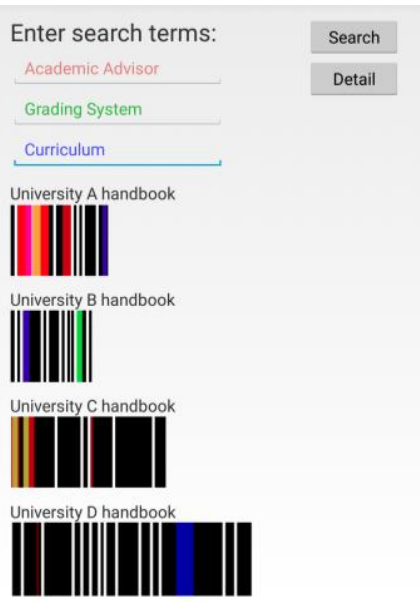

Fig. 1. An example image of the overview showing layouts and search term distributions in multiple text-based resources.

\section{Detail View}

The Detail View presents the user with a visualization that shows the underlying content of the selected areas of interest. To do this, a graph is generated, on the server which contains the text, using the Fruchterman-Reingold algorithm [11]. This image displays the search terms present within the selected sections, as well as what terms are related to them, and terms that are potentially related to them. Subterms we define to be a term that the author has specifically indicated in their index to be a term that is related to the search term. Potentially related terms we define as terms that share any number of pages, or 
are located within a certain proximity, with any of the search terms.

Each term in the graph is represented as a circle. The color of the circle corresponds to the colors from the Overview. Search terms are colored at full intensity of their corresponding color. Subterms are colored with a high intensity of the corresponding color, of the corresponding search term. Potentially related terms are colored with half of the intensity of their shared search term. If a potentially related term or subterm is shared between multiple search terms, the color of that node will be a combination of all of the aforementioned search terms' colors. The size of the circle is dependent on the relative amount of content contained in the selected sections, with a greater amount of content correlating with a larger circle. The result of this technique is shown in Fig. 2.

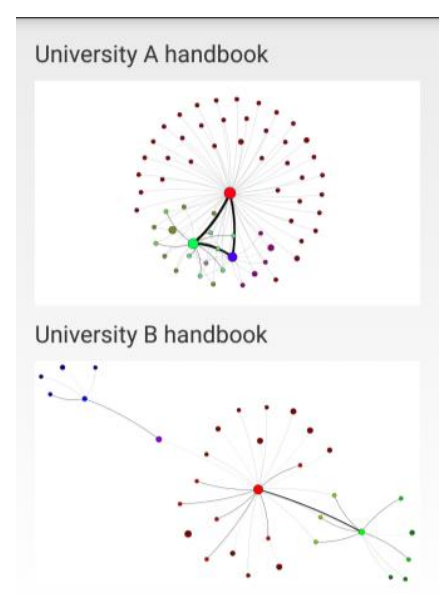

Fig. 2. An example image of the detail view showing frequency and relationship among search terms, related terms, and potentially related terms.

The terms are connected based off of the relationship between them. A search term to search term relationship is defined by whether or not the two terms share any number of pages, or are located within a certain proximity of each other. If they do share any number of pages, or are located in a predefined proximity of each other, a solid black line with varying thickness, based off the number of pages they share or closeness of their appearance, is drawn to connect them. For a search term to subterm relationship, a solid black line is drawn between them to indicate that the term is defined in the index as a related term. For a relationship of a search term to potentially related term, a gray line is drawn. To convey the relative strength of the relationship the line can have varying degrees of transparency, with a lower transparency correlating with a stronger relationship.

Zooming and panning is a functionality that is required on most mobile devices to optimize the usage of the provided screen space. After analyzing this information, the user can easily tell if the selected sections(s) are where they should look for the desired information. By activating a provided tool, the system will show the underlying text content for their review. This multi-level visualization approach will assist the user to review multiple entire documents intuitively, quickly identify potential areas of interest, and explore those contents through simple interactions.

\section{System Configuration}

In our proposed system, the documents, with associated table of contents and index, would be stored on a server, which would contain functionality for searching and selecting multiple documents for use with the visualizations. Additionally, this server would be responsible for generating the Detail View and transmitting it to the mobile device in a scalable vector graphics format. It is an XML-based file format that includes the description of the two-dimensional graphics objects in text-form. This facilitates fast and efficient transmission of visualized images between servers and mobile devices, as well as quick rendering on devices having limited computing power. Eclipse v4.2 Juno was the IDE used for the system development, utilizing the Android Developers Tools plugin. The device used to test our pilot design was a Nexus 5. The Nexus 5 has a 4.95" display, 2GB of RAM, and a $2.26 \mathrm{GHz}$ processor with 4 cores. Fig. 3 shows a picture of the MVI system showing the Overview visualization on the Nexus 5.

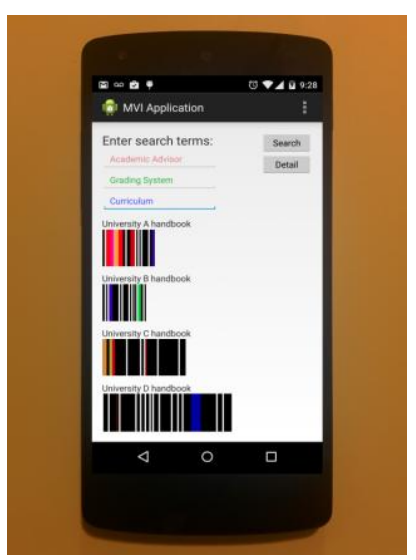

Fig. 3. Example image showing the overview visualization running on the Nexus 5.

\section{EXPERIMENTS}

To investigate the effectiveness of the MVI system for browsing lengthy text documents on mobile devices, survey-based usability tests were conducted to collect users' opinion of the visual interfaces when compared to a traditional text-based presentation system.

\section{A. Experimental Environment}

For the experiments, two testing applications were implemented on a desktop computer that simulated the browsing of text documents on small screen devices. Both applications present the same content with the same functionality differing only in presenting the document either in a text format or through visual abstractions as explained in Section II. Although both applications were uncomplicated and intuitive to use, each participant received an orientation on how to use both systems. The orientation explained the content viewing functionality provided by both systems and covered the visualization mechanism and meaning of the visual attributes used for the visual interfaces. Each user was then given enough time to investigate the two different viewing interfaces by browsing several text documents having different layouts and search term distributions.

Eight students majoring in computer science who were comfortable in the use of computers, had no problems with color perception, and had no prior experience with the 
developed visual interfaces participated in the experimental sessions.

After fully exploring both viewing interfaces, the participants completed a survey providing their feedback to different aspects of the MVI system. For an accurate evaluation of the proposed method, participants only evaluated the efficiency of the developed visual interfaces in reviewing text documents. The questionnaire used a 5 point Likert scale; 5 indicating the highest level of agreement, and 1 the lowest.

\section{B. Experimental Results}

Overall, participants expressed that the MVI system provides effective interfaces for searching and reviewing text documents. Regarding the visual interface that presents the overall view of the text content, evaluators strongly supported the effectiveness of the MVI system in: comparing multiple documents in standard search activities, inferring the layout of the documents, and identifying search term distributions in the text documents. All testers expressed that they either "Strongly agree" or "Agree" on all measured items associated with the Overview visualization.

Regarding the features associated with the Detail View visualization, which was designed for showing the underlying profile of selected areas of a text document, participants rated highly that the visualization delivered information about the frequency of terms associated with search terms and relationships among search terms, in the specified area of interest. A majority of testers "Strongly agree" that it is easy to identify terms that are related to multiple search terms in Detail View.

TABLE I: POST EXPERIMENT SURVEY RESULTS

\begin{tabular}{|c|c|}
\hline Questions & $\begin{array}{c}\text { Average } \\
(\text { Mean } \pm \text { SD) }\end{array}$ \\
\hline \multicolumn{2}{|l|}{ Related with Overview } \\
\hline $\begin{array}{l}\text { Easiness of comparing multiple text contents to identify } \\
\text { relevant resources }\end{array}$ & $4.75 \pm 0.46$ \\
\hline Easiness of understanding color coded visualization & $4.38 \pm 0.52$ \\
\hline Efficiency of relaying the layout information & $4.63 \pm 0.52$ \\
\hline Effectiveness of presenting search terms distribution & $4.5 \pm 0.53$ \\
\hline \multicolumn{2}{|l|}{ Related with Detail View } \\
\hline Intuitiveness of understanding color coded visualization & $4.38 \pm 0.74$ \\
\hline $\begin{array}{l}\text { Effectiveness of delivering frequency of terms associated } \\
\text { with search terms }\end{array}$ & $4.5 \pm 0.53$ \\
\hline Efficiency of delivering relationships among search terms & $4.63 \pm 0.52$ \\
\hline $\begin{array}{l}\text { Effectiveness of delivering relationships among } \\
\text { non-search terms }\end{array}$ & $4.25 \pm 0.71$ \\
\hline $\begin{array}{l}\text { Usefulness of sub-terms and associated information for } \\
\text { enhancing search activities }\end{array}$ & $4.13 \pm 0.64$ \\
\hline Efficacy of potential terms for exploratory search & $4.38+0.74$ \\
\hline $\begin{array}{l}\text { Easiness of identifying terms related with multiple search } \\
\text { terms }\end{array}$ & $4.75 \pm 0.46$ \\
\hline Overall efficiency of Detail View for enhancing search & $4.0 \pm 0.53$ \\
\hline \multicolumn{2}{|l|}{ Related with Entire System } \\
\hline Overall efficiency of MVI as a content browsing tool & $4.5 \pm 0.53$ \\
\hline Preference of using multi-level visualizations & $4.63 \pm 0.52$ \\
\hline Overall inclination of using MVI over text-based system & $4.25 \pm 0.89$ \\
\hline
\end{tabular}

For both visualizations, evaluators commented that they did not experience major difficulties regarding understanding the meaning of visual attributes. Participants considered that the visual interface providing an overview of the information is more effective than the visualization presenting the underlying content of a selected area. Testers also responded very positively to other visualization features, but not as strongly as compared to other measures, such as presenting relationships among non-search terms, subterms, and terms presented for exploratory search. Our post experimental analysis found that the Detail View visualization may display too much information on a limited screen space, which may cause information overloading to the participants. This is one of the tasks that we should address in the future.

In general, testers concluded that the developed visual interfaces are effective in browsing text contents and the multi-level visualization assisted their decision making process. Although participants only experienced these visual interfaces in searching text documents for the first time, $75 \%$ of testers would prefer to use our developed visualization over a standard text-based system, while the remaining $25 \%$ of testers didn't have any specific preference. Table I shows the summation of the survey results, summarizing all user feedback for the MVI system in comparison to a text-based presentation system. The questions are grouped in three different major perspectives: Overview, Detail View, and the entire system.

\section{DISCUSSION}

The proposed method uses the table of contents and index for generating the graphical illustration of a given text document. The table of contents is used to succinctly show the length and structure of a given material. The index is used primarily as a means to present more specific information, such as location of search terms and related terms in the text. Lengthy text documents are commonly accompanied with a table of contents and index, if this is the case then the developed method is immediately applicable with little to no modifications. If this supporting information is not available, then the proposed approach can be applied after generating the table of contents and index via parsing. Methodology for generating a table of contents and index should be included to address this issue. Since the designed visualizations rely heavily on these two elements; a man-made table of contents and index will produce more meaningful output, as they are more informative than ones procedurally generated. The proposed visualization technique can be easily applied to other data domains that have a large amount of text contents.

The Overview provides the user with a general view of the structure and term distribution of the given materials for the purpose of comparison and contrast. The Detail View, in tandem, presents the desired underlying information at any given section of the shown texts. By presenting information in this two-tiered fashion, our system will assist users to review the lengthy content effectively; even with the limited screen space. Through both of these things it is easy to see what areas contain the information needed. Since the Detail View is dependent upon user selections from the Overview, the information needing to be displayed in the Detail View is significantly reduced. This is especially useful on these devices which can be unwieldy to utilize effectively for 
displaying large amounts of text-based data. In addition, the separation of both the Overview and Detail View can significantly reduce the workload on the user and further increase their comprehension of the information displayed.

Another limitation that we had to address was the lower transmission rates for data. It can be difficult to fully display the desired information while dealing with a fairly low bandwidth. To address this, only the selected areas that the user has deemed of interest will be transmitted to the device. This way, the need to send large amounts of data is avoided. The result of this is that our technique shows a large amount of information through the visual abstractions, thus compacting the large amount of information that needs to be displayed, and then only shows the desired text information when needed.

\section{FUTURE WORK}

Despite the shown merit to our approach there are several areas in which it can be enhanced. Providing more text information, through tooltips or other tools; that can be used simultaneously with the visualization, might be one way to provide additional information that may not be covered from just the visualizations. Additionally, a tool to zoom, for both visualizations, should be implemented to combat the inherent issues that arise with small screens. Another potential enhancement of the Overview may be to make exceedingly long documents wrap, or translate them into boxes to more effectively make use of the space available.

The Java Universal Network/Graph Framework was utilized to generate the graph used in the Detail View. An issue with this is that the distance between circles, representing each term, in the Detail View is not utilized. In order to present circles without much overlapping, the layout algorithm can't specify distance among circles, which is one of the instinctive visual attributes to represent relationships between terms. Developing a new algorithm that can utilize that distance between them, while avoiding significant overlapping between them, will be an important future task to further enhance the information provided. This is especially important since every pixel matters to compactly display the results.

Techniques such as stemming, or utilizing synonyms, can also be a boon to the visualization techniques. Stemming can allow us to combine similar terms from the same root into one, which will simplify the Detail View where several variations of a given term are represented as multiple circles. This inevitably increases the complexity of the visualization. In this same vein of thinking, utilizing synonyms can further compact the visualization and reduce the complexity. Additionally, this way the user does not have to enter precisely the exact term needed to generate the desired output.

Our technique does not only have to be limited to documents, further improvements can be implemented to generalize this technique to apply to a multitude of other information that is available on the Internet. For example, in the future, the proposed visualization techniques can be applied to compare results from online shopping. This way it makes it easier to evaluate many items at once, as well as to only browse items that are related to the user's interest.
Further, we can utilize this technique with various forms of social network information such as blogs, discussion forums, etc. With these visualizations a user can easily skim through a large amount of information and quickly identify valuable posts including their interests.

\section{CONCLUSION}

We have introduced a technique for visualizing lengthy text-based documents on mobile devices that lack screen space. The user can get visualized images presenting multiple materials' structures, query for distributions within the documents, and then pinpoint areas that they are interested in. Visual representations of information, at different levels of detail, are closely intertwined, so that users can navigate a large information workspace towards specific locations. They will then be provided with the information contained within, which is useful for further analysis, exploratory search, or query refinement. The overall process streamlines the approach of having to scroll through very long documents on a small device, saving the user time and effort. Our pilot study demonstrated the viability of the MVI system for browsing text content on mobile devices. The experimental survey shows that visualization enhanced content browsing is well-liked by users over a traditional text-based presentation. This study also suggests a future task to better present lengthy text documents on small screen devices more effectively. Finally, the technique presented here can serve as a baseline for further improvement regarding effectively viewing other fast growing information that is available on the Internet and various social networks.

\section{REFERENCES}

[1] B. Kim and B. Aeschliman, "A technique for creating visually abstracted geometric thumbnails of web documents for mobile devices," International Journal of Digital Content Technology and Its Applications, vol. 6, no. 13, pp. 470-481, July 2012.

[2] V. Roto, A. Popescu, A. Koivisto, and E. Vartiainen, "Minimap: A web page visualization method for mobile phones," in Proc. the SIGCHI Conference on Human Factors in Computing Systems, 2006, pp. 35-44.

[3] A. Cuttone, S. Lehmann, and J. Larsen, "A mobile personal informatics system with interactive visualizations of mobility and social interactions," in Proc. the 1st ACM International Workshop on Personal Data Meets Distributed Data, 2013, pp. 27-30.

[4] N. Correia, C. Lopes, J. Hawkey, S. Oliveira, and O. Perriquet, "Personal routine visualization using mobile devices", in Proc. the 11th International Conference on Mobile and Ubiquitous Multimedia, 2012.

[5] M. Chen, C. Chen, S. Liu, and K. Zhang, "Mobile visualization supporting awareness in collaborative software development," in Proc. the 7th International Symposium on Visual Information Communication and Interaction, 2014, pp. 123-130.

[6] D. Zhang, G. Karabatis, Z. Chen, B. Adipat, L. Dai, Z. Zhang, and Y. Wang, "Personalization and visualization on handheld devices," in Proc. the 2006 ACM Symposium on Applied Computing, 2006, pp. 1008-1012.

[7] S. Huot and E. Lecolinet, "Spira list: A compact visualization technique for one-handed interaction with large lists on mobile devices," in Proc. the 4th Nordic Conference on Human-Computer Interaction: Changing Roles, 2006, pp. 445-448.

[8] C. Tseng, M. Ali, and R. Vibhandik, "Common visual representation for websites and smartphones," in Proc. the 2010 IEEE Conference on Granular Computing, 2010, pp. 477-482.

[9] A. Abubakar, A. Zeki, and H. Chiroma, "3D mobile map visualization concept for remote rendered dataset," in Proc. the 2nd International 
Conference on Advanced Computer Science Applications and Technologies, 2013, pp. 228-231.

[10] G. Short and B. Kim, "Multi-tiered visual interfaces for book search with digital library systems," in Proc. the 6th International Conference on Multimedia, Computer Graphics, and Broadcasting, 2014, pp. 21-23.

[11] T. Fruchterman and E. Reingold, "Graph drawing by force-directed placement," Software-Practice \& Experience, vol. 21, no. 11, pp. 1129-1164, Nov. 1991.

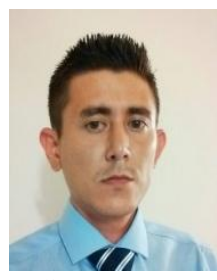

Todd Welch is currently a computer scientist at the Naval Surface Warfare Center (NSWC) in Crane, Indiana, USA. Mr. Welch obtained his master's degree in computer science, in the spring of 2015 from Indiana University-Purdue University Fort Wayne, Indiana, USA. He is a member of Upsilon Pi Epsilon. His current research interests are mobile application development, computer graphics, and augmented

reality.

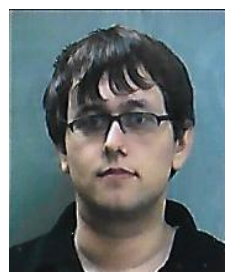

Gregory Short is a graduate student of computer science at the University of Wisconsin-Madison. Mr. Short obtained his bachelor's degree in computer science, in the spring of 2015 from Indiana University-Purdue University Fort Wayne, Indiana, USA. He is a member of Upsilon Pi Epsilon. His current research interests are data visualization, computer graphics, artificial intelligence, and machine

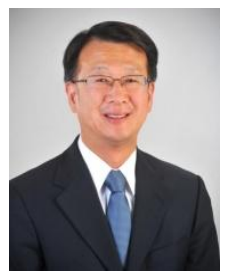

Beomjin Kim is the director of Information Analytics and Visualization Center and a professor of computer science at Indiana University-Purdue University Fort Wayne, Indiana, USA. Dr. Kim obtained his Ph.D. in computer science from the Illinois Institute of Technology in 1998. He received several research awards including the Distinguished Research Award from Allied Academies and Researcher of the Year Award from IPFW Sigma Xi Chapter, conducted projects funded by National Science Foundation, Purdue University, regional business, and serves as a member of editorial board or review board on journals such as the International Journal of Computational Science and The International Journal of Digital Contents and Applications. His current research interests include data visualization, 3D vision technology, HCI, and computer graphics. 